\title{
Increased phosphorylation on residue S795 of the retinoblastoma protein in esophageal adenocarcinoma
}

\author{
AKUENI L. DAVELAAR ${ }^{1,2}$, DANIELLE STRAUB ${ }^{1,2}$, KAUSHAL B. PARIKH $^{1,2}$, \\ LIANA LAU $^{1,2}$, PAUL FOCKENS $^{1}$ and KAUSILIA K. KRISHNADATH ${ }^{1,2}$ \\ ${ }^{1}$ Department of Gastroenterology and Hepatology, and ${ }^{2}$ Center for Experimental and Molecular Medicine, \\ Academic Medical Center, Amsterdam, The Netherlands
}

Received November 12, 2014; Accepted January 5, 2015

DOI: 10.3892/ijo.2015.3040

\begin{abstract}
Due to its increasing incidence and relatively poor prognosis, esophageal adenocarcinoma (EAC) is becoming a significant health problem. Elucidating the mechanisms underlying EAC development is of great importance to improve upon current conventional treatment strategies. Insight into phosphorylation has proven to be useful for the development of diagnostic and molecular treatment strategies in cancer. A pathway largely dependent on phosphorylation and frequently deregulated in cancer is the cell cycle regulating p16-retinoblastoma $(\mathrm{Rb})$ pathway. We investigated kinase activity, specifically phosphorylation within the p16-Rb pathway, in EAC. A high-throughput peptide tyrosine kinase array containing short peptides representing 100 proteins with known phosphorylation sites, was used to assess phosphorylation activity in EAC. Also, specific phosphorylation changes of the cell cycle protein $\mathrm{Rb}$ and its upstream regulator P16 were validated through immunoblotting in EAC and normal esophageal cells and tissues. Phosphorylation activity was higher in EAC tissues as compared to normal squamous esophageal tissues. A majority of the proteins significantly higher phosphorylated in EAC were found to be involved in cell structure maintenance and immunity. Validation of $\mathrm{Rb}$ phosphorylation in EAC biopsy specimens and cell lines showed hyper phosphorylation of $\mathrm{Rb}$ associated with aberrant $\mathrm{P} 16$ expression in the cancer tissues. The specific Rb (S795) residue was significantly higher phosphorylated in EAC compared to normal esophageal tissue (Wilcoxon paired rank test, $\mathrm{P}=0.004)$. Investigation of $\mathrm{Rb}(\mathrm{S} 795)$ phosphorylation may indicate targets for intervention and give more molecular insight in EAC.
\end{abstract}

Correspondence to: Professor Kausilia K. Krishnadath, Department of Gastroenterology and Hepatology, Academic Medical Center, Room C2-111, Meibergdreef 9, 1015 AZ Amsterdam, The Netherlands

E-mail: k.k.krishnadath@amc.uva.nl

Key words: esophageal adenocarcinoma, phosphorylation, retinoblastoma protein, $\mathrm{p} 16$

\section{Introduction}

For a number of decades the incidence of esophageal adenocarcinoma has been steadily rising in several countries (1-4). In the USA, for instance, the overall EAC incidence increased from 0.4 cases per 100,000 in 1975 to 2.6 cases per 100,000 in 2009 (5). The 5-year survival has improved in this same period from $\sim 5 \%$, but still remains rather poor at $15-20 \%(3,5)$. The exact reasons for this rise in EAC incidence is unclear. With respect to risk factors some studies have shown a relationship between increased body mass index (BMI) with an increased risk for EAC $(3,6)$. Other important risk factors for EAC are gastro-esophageal reflux disease (GERD) and the herewith related Barrett's esophagus (BE) (7-9). BE is a metaplastic pre-malignant transformation of the esophageal epithelium associated with GERD (7-9). Although the outcomes of EAC have improved through the bettering of conventional treatments, for instance through combining chemoradiotherapy with surgery, outcomes of EAC are still poor (10). There is, thus, a need for a deeper molecular insight into EAC to develop new approaches and treatments to improve patient outcomes.

A potential route to this deeper molecular insight are kinases. This group of enzymes, through addition of a phosphate group to specific targets, activate large and far-reaching signaling cascades, which are pivotal in maintaining homeostasis in living organisms. Their pivotal role also means that the precarious biological balance they help sustain can be easily broken, through the mutation or aberrant expression of these bio-molecules. Elucidating phosphorylation activity with respect to specific processes, such as cell proliferation, has been shown to be an attractive avenue of research as molecular strategies developed around inhibitors of the involved kinases might serve as anticancer therapeutics. For instance, the targeting of the HER2 receptor tyrosine kinase has been shown to be of benefit in breast cancer treatment $(11,12)$. Also in EAC, amplification of the HER2 gene has been noticed in up to $21 \%$ of samples (13). A recent trial has shown improvement in survival of patients with gastric and gastroesophageal junction cancer upon combining chemotherapy with the HER 2 targeting immunoglobulin trastuzumab (14). Therefore, identifying novel aberrancies of kinases involved in cell proliferation could be useful in EAC. A potential source for these kinases is 
the p16-Rb pathway, which is frequently affected in cancer (15). The P16/INK4A protein inhibits the cyclin D-CDK4/6 complexes that modulate progression through the G1/S-phase checkpoint of the cell cycle, through hyperphosphorylation of the Rb-E2F complex. Release of the E2F transcription factors consequently leads to DNA replication (15). Aberrancy of p16, such as mutations, methylations and deletions, are some of the earliest events in cancer (16). P16 is also involved in the pathogenesis of EAC with a number of P16 alterations such as gene locus loss, LOH (loss of heterozygosity), mutations and methylation being present in $\mathrm{BE}$, the pre-malignant stage of EAC (17-20) and consequently increasing with increasing tissue dysplasia $(17,18,20)$. In a number of cancers it has been shown that aberration of p16 affect phosphorylation status of $\mathrm{Rb}(21,22)$. It would be of interest to investigate whether a similar biomolecular effect can be seen in EAC.

To elucidate phosphorylation mechanisms involved in the pathogenesis of EAC we first employed a high-throughput tyrosine kinase peptide chip, to investigate broad-scale kinase activity in EAC. We additionally examined the $\mathrm{p} 16-\mathrm{Rb}$ pathway in EAC and normal squamous esophageal biopsy specimens to investigate whether phosphorylation of $\mathrm{Rb}$ might play a role in EAC pathogenesis.

\section{Materials and methods}

Patient material. The present study received Institutional Review Board approval from the Academic Medical Center (AMC, Amsterdam, The Netherlands) and written informed consent was obtained from all participating subjects. Biopsies were obtained from EAC patients referred for endoscopy for disease staging. The study included biopsies of EAC and squamous mucosa as confirmed in matched samples taken for routine histological purposes. For the kinase chip, immunoblotting and immunohistochemistry experiments normal squamous EAC tissue samples were isolated from a total of 51 patients. Samples were snap frozen and stored at $-80^{\circ} \mathrm{C}$ until processing for experiments. Of the patients, 43 were male and 8 female. Average age was $66 \pm 12$ years.

Esophageal cell lines. The human hTERT immortalized esophageal cell line EPC2-hTERT was a kind gift from Professor A. Rustgi (University of Pennsylvania, Philadelphia, PA, USA) (23) and was cultured in KSFM medium (Life Technologies, Bleijswijk, The Netherlands) supplemented with human recombinant epidermal growth factor (EGF) and bovine pituitary extract (Life Technologies).

The OE19 and OE33 esophageal adenocarcinoma cell line were obtained from the American Type Culture Collection (ATCC; Manassas, VA, USA) and cultured in RPMI-1640 medium (Life Technologies) supplemented with L-glutamine, penicillin/streptomycin and $10 \%$ fetal bovine serum (FBS; Lonza Group AG, Basel, Switzerland).

Tyrosine kinase peptide array. Fifteen EAC and coupled squamous biopsies were analysed with the PAMgene tyrosine kinase peptide-microarray system (PAMgene, Hertogenbosch, The Netherlands) for two replicate experiments. This peptidemicroarray contains 143 short peptides, with sequences derived from literature, with known phosphorylation sites representing
100 proteins. Biopsies were lysed in M-PER lysis buffer (Thermo Fisher Scientific, Etten-Leur, The Netherlands) and samples were tested in 2-3 replicates. The peptide-microarray system measures kinase kinetics by detecting phosphorylation with anti-phospho tyrosine fluorescently labelled immunoglobulins. End level average signals and initial rates of the samples were determined and corrected for signal saturation. These measurements indicated phosphorylation activity. Kinase activity heat maps were created with the CIMminer software (Genomics and Bioinformatics group, LMP, CCR, National Cancer Institute, http://discover.nci.nih.gov/cimminer/).

DNA fluorescence in situ hybridization (FISH). To detect the loss of the p16 gene locus in the cell lines, DNA FISH was performed as previously described (24). For this experiment the specimens were hybridized with a directly labelled probe mix which contained the SpectrumRed-LSI p16 (9p21) locus specific probe for the 9p21 (P16) region on chromosome 9, obtained from Abbott Molecular (Chicago, IL, USA).

Scoring was done with the 60x objective of the Olympus BX51 microscope (Olympus Nederland BV, Zoeterwoude, The Netherlands). Pictures were taken with the Olympus XM10 camera and brightness and contrast adjustments were done with Olympus Cell^F software. To define per sample P16 DNA-FISH status we used previously determined frequency cut-off values for P16 gains and losses (24). These values were obtained from counts of 20 normal squamous epithelium cytological patient specimens (100 nuclei evaluated per case) and calculated as the mean percentage of squamous nuclei with either $p 16$ gain or loss plus $3 \mathrm{x}$ times standard deviation. The P16 cut-off frequency was $10 \%$ for losses and $4 \%$ for gains.

Western blotting. Biopsies and cell lines were lysed in M-PER lysis buffer (Thermo Fisher Scientific) supplemented with protease and phosphatase inhibitors (Halt protease and phosphatise inhibitor cocktail; Thermo Fisher Scientific). For the cell line experiments two independent lysate samples were made from each line and a number of replicate blots were made from these samples.

Lysates combined with sample buffer $(125 \mathrm{mM}$ Tris- $\mathrm{HCl}$, pH 6.8; $4 \%$ SDS; $2 \% \beta$-mercaptoethanol; $20 \%$ glycerol; $1 \mathrm{mg}$ bromophenol blue) were loaded onto SDS-protein gels and subsequently transferred onto PVDF membranes (Millipore, Amsterdam, The Netherlands). The blots were incubated overnight at $4^{\circ} \mathrm{C}$ with the primary antibody of interest. After incubation with the primary antibodies, blots were incubated for $1 \mathrm{~h}$ at room temperature with the secondary antibody, the anti-rabbit HRP conjugated immunoglobulin (Dako, Heverlee, Belgium). Consequently blots were incubated in Lumilite plus (Boehringer-Mannheim, Mannheim, Germany) and chemiluminescence was detected using a Fuji LAS 4000 illuminator (Fuji Film Medical Systems, Stamford, CT, USA). Quantification of the blots was performed with ImageJ software (version 1.44). Primary antibodies used were the rabbit anti-phospho-Rb (T356), anti-phospho-Rb (S795) and antiphospho-Rb (S780) immunoglobulins (Abcam, Cambridge, UK). Also used were the rabbit monoclonal anti-P16/INK4A (Epitomics/Bio-Connect BV, TE Huissen, The Netherlands) and rabbit polyclonal anti-Rb (ab6075; Abcam) antibodies. The rabbit polyclonal anti-actin (Santa Cruz Biotechnology, 
Heidelberg, Germany) antibody was used for quantification of the P16 and (phospho-)Rb levels.

The relative phospho- $\mathrm{Rb}$ levels were corrected for the total level of the $\mathrm{Rb}$ protein to determine the phosphorylation activity on the specific amino acid. Total $\mathrm{Rb}$ levels were determined for each cell line and EAC and squamous patient biopsies. From these levels an average $\mathrm{Rb}$ level was determined for each of the aforementioned esophageal cell lines and the BE tissue types. The average phospho- $\mathrm{Rb}$ levels were then corrected for these total $\mathrm{Rb}$ levels for the respective cell line and tissue type.

For the tissue samples total $\mathrm{Rb}$ expression was obtained for 24 squamous and 20 EAC samples. Phospho-Rb levels were measured for 38 normal squamous and 32 EAC samples. Phospho-Rb (S795), was investigated in protein lysates from 12 EAC and squamous samples. Phospho-Rb (S780) was investigated in $4 \mathrm{EAC}$ and squamous samples. Phospho-Rb (T356) was investigated in 16 EAC and 22 squamous samples. P16 protein levels were measured for 30 normal squamous and 20 EAC samples.

Immunohistochemistry. Eight squamous esophageal and 19 EAC samples were used for the immunohistochemistry experiments. These samples were formalin fixated after isolation and paraffin embedded. Subsequent $5-\mu \mathrm{m}$ tissue sections of each biopsy were cut and used for the stainings. For staining, tissue slides were de-paraffinized and antigen retrieval was performed by boiling slides for $20 \mathrm{~min}$ in pre-warmed citrate buffer, $\mathrm{pH}$ 6.0. Endogenous peroxidase was blocked by incubation with $0.3 \% \mathrm{H}_{2} \mathrm{O}_{2}$. Next, slides were blocked with $10 \%$ normal goat serum (Bio-Connect) and an avidin/biotin blocking kit (Vector Laboratories Inc., Burlingame, CA, USA). Slides were washed in PBS and incubated with the primary antibody for $90 \mathrm{~min}$ at room temperature. Primary antibodies used were monoclonal rabbit anti phospho- $\mathrm{Rb}$ (T356) and polyclonal rabbit anti phospho-Rb (S795) (Abcam). Slides were then incubated with the respective biotin linked secondary reagents from the LSAB ${ }^{\mathrm{TM}} 2$ Kits (Dako) following the manufacturer's instructions. The peroxidase activity was visualized using $\mathrm{DAB}^{+}$(Dako). Finally, sections were counterstained with Mayer's haematoxylin, dehydrated and mounted. Slides were photographed with a microscope equipped with a digital camera (Leica CTR500; Leica Microsystems, Rijswijk, The Netherlands) using the 10x objective.

A semi-quantitative approach was used to score the stained tissue samples taking into account the strength of staining and the nuclei stained. Only nuclei of epithelial cells were considered for scoring. For the anti-phospho-Rb (T356, S795) antibodies, staining was scored from 0 to 4 . Scores 0,1 , no or very light staining in few nuclei; score 2 , staining in few nuclei; score 3, staining in 33-50\% of nuclei; score 4, clear staining in almost all nuclei. Slides were scored by one researcher (D.S.).

Statistical analysis. The Paired t-test and the Wilcoxon paired-rank test to determine peptides significantly differently phosphorylated in tumor compared to normal tissues were performed in Microsoft Excel and with SPSS 20 (IBM, Amsterdam, The Netherlands). Statistical significance was set at $\mathrm{P}<0.05$. The T-test, the Wilcoxon paired rank and the Mann-Whitney $U$ test to look for significant differences in quantified protein expression were performed with GraphPad Prism 5 (GraphPad Software Inc., La Jolla, CA, USA). Statistical significance was set at $\mathrm{P}<0.05$. SEM of the quantified protein expressions was also determined with GraphPad.

\section{Results}

Tyrosine kinase activity and phosphorylation of the Rb protein in esophageal adenocarcinoma. Phosphorylation activity in EAC biopsies was compared to the matching normal squamous samples for 15 patients with the PAMgene tyrosine kinase peptide-microarray system in two independent experiments. In both experiments phosphorylation activity showed an overall increase in tumor samples compared to the matching normal squamous biopsies (Fig. 1). For one experiment, phosphorylation levels were too low to be analysed for all the peptides. In this case there were 100 analysable peptides. For the other experiment 142 peptides were analysable. When averaged over both experiments, of the 142 analysable peptides, $122(86 \%)$ were more phosphorylated and $11(8 \%)$ were less phosphorylated in EAC. Nine (6\%) peptides had equal phosphorylation levels in tumor compared to normal squamous tissues. In both experiments we found a number of peptides for which the difference in phosphorylation between tumor and coupled normal squamous tissues was significant $(\mathrm{P}<0.05$; paired t-test and Wilcoxon paired rank test). We listed all peptides that were either significantly differently phosphorylated for tumor vs. normal tissues in both experiments or in one experiment if it was only analysable in a single experiment. These peptides can be assigned to proteins which have a function in a variety of processes including development, immunity and also cell structure and motility (Table I).

We found that also peptides representing proteins of the G1/S checkpoint, specifically CDK2 and Rb, were differently phosphorylated in EAC vs. normal esophageal tissues. Both peptides showed higher levels of phosphorylation in tumor vs. normal tissues, with ratios of 1.7 and 1.5 , respectively for CDK2 and $\mathrm{Rb}$. Notably, phosphorylation of the CDK2-peptide was on a presumed inhibitory residue at the Y15 position (25), which should lead to a lower level of CDK2 activity. Nonetheless, the peptide derived from the $\mathrm{Rb}$ protein showed higher phosphorylation in EAC compared to the normal tissues. This is most likely due to a larger contribution of CDK4 activity, resulting in a net effect of increased $\mathrm{Rb}$ phosphorylation in EAC.

P16 gene status and protein expression in normal squamous and malignant esophageal cell lines. To investigate in cell lines if increased levels of $\mathrm{Rb}$ phosphorylation were associated with p16 abnormalities in EAC, we first validated gene status and the relative protein expression of P16 in cell lines representing normal squamous epithelium (EPC2-hTERT) and EAC (OE19 and OE33). The P16 (9p21) gene locus status of the cells was investigated through DNA FISH (Fig. 2) and protein expression of P16 by immunoblotting. EPC2-hTERT was considered normal for P16 gene status as assessed by DNA-FISH (Table II). Both EAC cell lines had large frequencies of P16 FISH abnormalities. OE19 showed cells with either losses or gains of the P16 allele, while OE33 exhibited exclusively gains of the gene (Table II). When considering the protein levels, EPC2-hTERT had the highest relative 

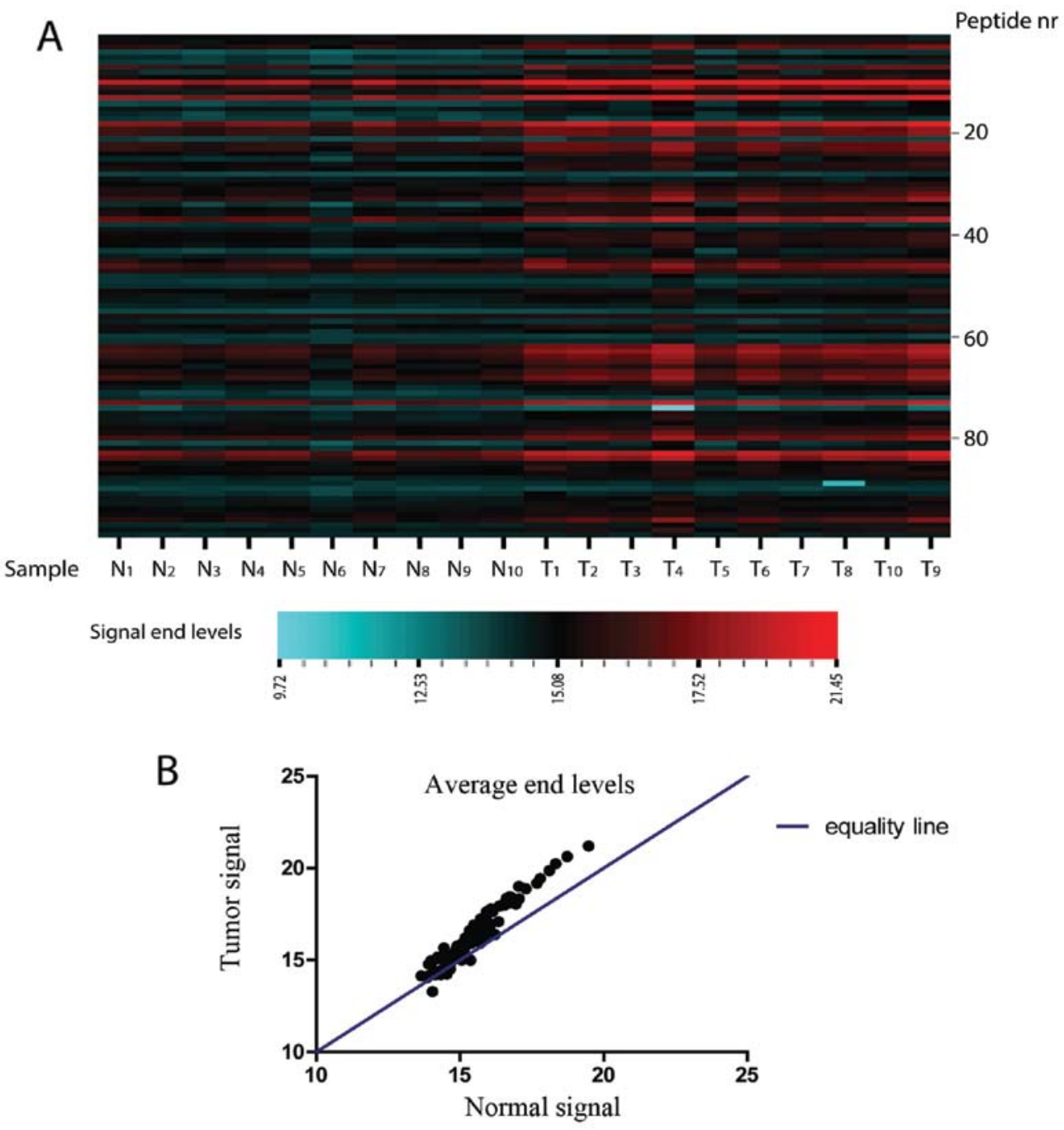

Figure 1. (A) Heatmap of end-level phosphorylation signals for 7 esophageal adenocarcinomas ( $\mathrm{T}$ ) and matching normal squamous samples (N) for 100 peptides of the PAMgene tyrosine kinase chip. Samples 1, 4 and 6 were excluded from further analysis as histopathology indicated the tumor samples not to be esophageal adenocarcinoma. (B) Average end-level phosphorylation signals. Peptides above the equality line have higher phosphorylation levels in the tumor compared to the matched normal squamous samples.

Table I. Proteins with significantly different $(\mathrm{P}<0.05)$ phosphorylation signals in tumor $(\mathrm{T})$ compared to normal squamous $(\mathrm{N})$ esophageal tissues as measured by the kinase array.

\begin{tabular}{|c|c|c|}
\hline Pathway & Protein (gene) - [p-Tyr group] & Fold-change (T/N) \\
\hline \multirow[t]{4}{*}{ Cytoskeletal structure/cell motility } & Annexin A2 (ANXA2) - [Y24] & 4.9 \\
\hline & Adapter molecule Crk $(C R K)$ - [Y221] & 3.0 \\
\hline & Macrophage-stimulating protein receptor $(R O N)$ - [Y1353] & 2.4 \\
\hline & $\beta$-catenin $(C T N B 1)$ - [Y86] & 1.9 \\
\hline \multirow[t]{2}{*}{ Development/homeostasis } & Tyrosine-protein kinase JAK2 (JAK-2) - [Y570] & 1.6 \\
\hline & Myelin basic protein $(M B P)-[Y 203]$ & 1.5 \\
\hline \multirow[t]{7}{*}{ Immunity } & T-cell surface glycoprotein CD3 $\zeta$ chain $(C D 3 Z)-[Y 123,153]$ & $4.0,5.5$ \\
\hline & Signal transducer and activator of transcription 4 (STAT4) - [Y725] & 4.0 \\
\hline & B-cell antigen receptor complex-associated protein $\alpha$-chain $(C D 79 A)$ & \\
\hline & [Y182/188] & 1.6 \\
\hline & Lymphocyte cell-specific protein-tyrosine kinase ( $L C K)$ - [Y394] & 1.4 \\
\hline & Linker for activation of T-cells family member $1(L A T)$ - [Y255] & 1.2 \\
\hline & Phosphoinositide phospholipase C- $\gamma-1$ (PLCG1) - [Y1253] & 0.63 \\
\hline
\end{tabular}

Proteins are collected according to the biological roles most prominently connected to the phospho-group of interest. 


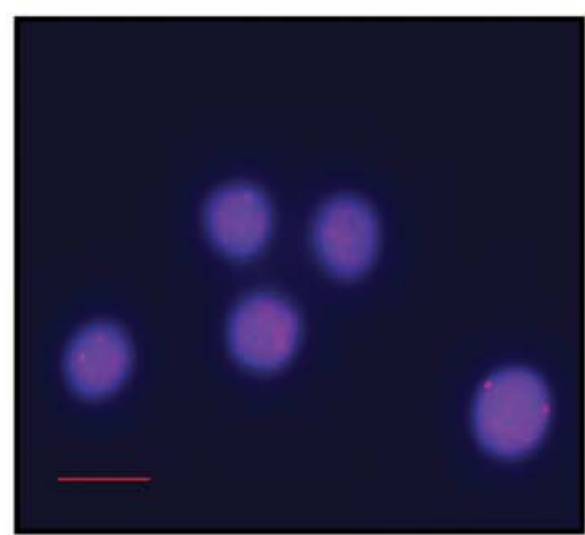

EPC2-hTERT

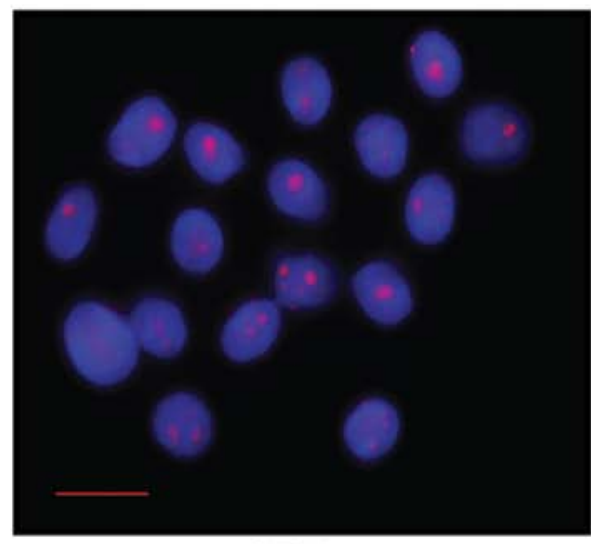

OE19

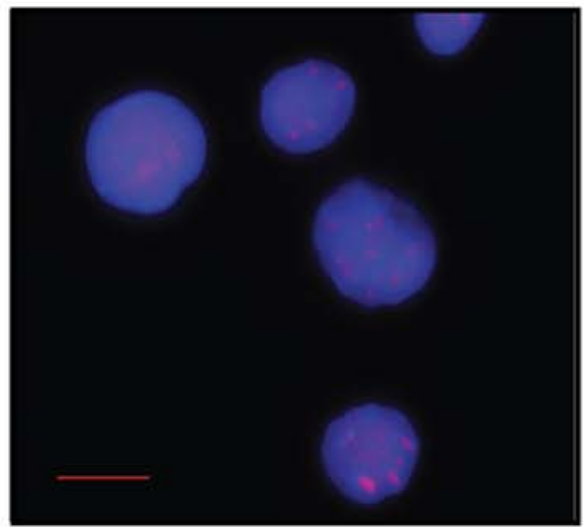

OE33

Figure 2. DNA FISH results with LSI p16 probe on normal squamous EPC2-hTERT, OE19 and OE33 esophageal adenocarcinoma cell lines. Red signals indicate the p16 (9p21) gene loci. Cell nuclei are counterstained with DAPI. Scale bar indicates $15 \mu \mathrm{m}$.

p16 protein expression. In comparison, the cancer cell line OE33 which actually showed gains of P16, had a lower P16 protein expression, while OE19 had the lowest expression level (Table II and Fig. 3A). The low protein levels of P16 in OE33 is likely due to $P 16$ gene promoter hypermethylation which is a frequent event in EAC and also has been observed in OE33 (26). Thus, P16 protein levels correlated with $P 16$ gene status as assessed by DNA-FISH in EPC2-hTERT and OE19. This correlation was not directly seen for OE33. Nevertheless, both techniques indicated aberrancies of p16 in cancer cells
Table II. Frequencies for p16 locus loss and gain in esophageal cell lines by DNA FISH.

\begin{tabular}{lccc}
\hline Cell lines & EPC2-hTERT & OE19 & OE33 \\
\hline P16 FISH frequencies (\%) & & & \\
Loss & 0 & 13 & 0 \\
Gain & 1 & 24 & 68 \\
\hline
\end{tabular}

Nuclei (100) were counted for all cell lines. OE19 and OE33 are esophageal adenocarcinoma cell lines. EPC2-hTERT is a normal squamous esophageal cell line.

compared to normal squamous cells. However, the assessed P16 protein levels in EAC seemed to correlate better with the reported functionality of the protein.

Phospho-Rb expression in normal and EAC cell lines. Consequently, we compared the P16 protein expression levels to the relative $\mathrm{Rb}$ protein phosphorylation in the EPC2-hTERT, OE19 and OE33 cell lines. Rb has several phosphorylation sites. We investigated phosphorylation on the T356 and S795 amino acids of $\mathrm{Rb}$ in the cell lines. We found that on average $\mathrm{Rb}$ phosphorylation on these two residues inversely correlated with the P16 protein levels (Fig. 3). P16 protein levels were lower for both EAC cell lines, OE19 and OE33, compared to the normal squamous cell line, EPC2-hTERT (Fig. 4A). For the T356 residue, EPC2-hTERT, showed a lower phospho-Rb (T356) level compared to the EAC cell line OE19 and comparable levels to OE33. For phospho-Rb (S795) a higher level of phosphorylation was seen for both EAC cell lines as compared to the normal squamous cell line.

$R b$ phosphorylation and P16 expression in esophageal tissues. $\mathrm{Rb}$ protein phosphorylation was also investigated in patient biopsies with EAC and normal squamous epithelial tissues and compared with P16 protein expression (Fig. 4). For these samples phosphorylation on the S795, S780 and T356 amino acids of Rb was investigated. In all cases, the tissue phospho-Rb levels were corrected for total Rb expression. The protein expression levels within the specific tissue types were rather variable (Fig. 4B-D). On average, expression of P16 was decreased in EAC biopsies when compared to the normal squamous esophageal tissues (Fig. 4B, t-test; $\mathrm{P}=0.049$ ). Inversely, we found increased phosphorylation for all three investigated $\mathrm{Rb}$ sites in EAC as compared to normal squamous tissues (Fig. 4D). On average, Rb phosphorylation at the S795 site was significantly higher in EAC compared to coupled squamous tissues (Wilcoxon paired rank test, $\mathrm{P}=0.004)$. Considering the specific phosphorylation sites, for phospho-Rb (S795), 9 of 12 (75\%) of EAC cases showed increased phosphorylation as compared to matching squamous controls. For phospho-Rb (S780), 4 of 4 (100\%) and for phospho-Rb (T356), 8 of 16 (50\%) had increased phosphorylation in EAC. The inverse correlation between a higher $\mathrm{Rb}$ phosphorylation ratio compared to P16 protein expression in EAC falls in line with the functional relation of P16 and Rb phosphorylation. 
A
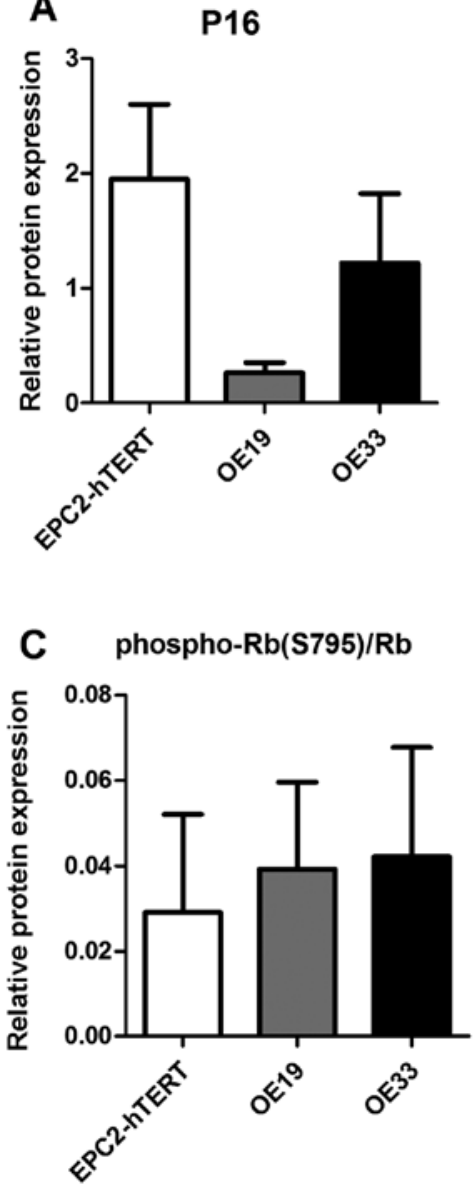

B

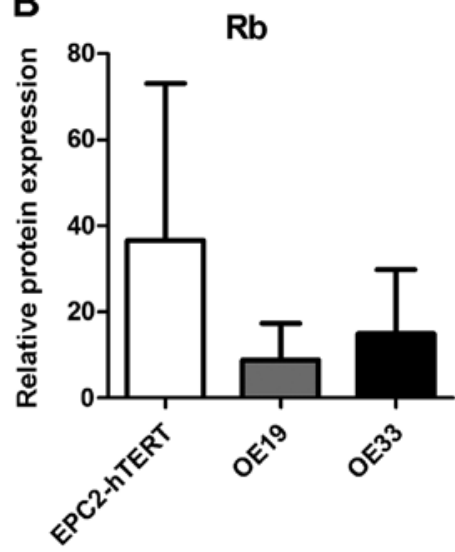

D phospho-Rb(T356)/Rb

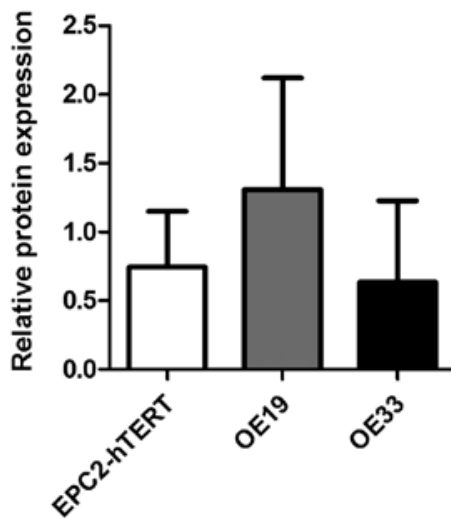

Figure 3. Quantification of immuno-blots of esophageal cell line lysates for p16 and Rb phosphorylation. All cases were corrected for actin that was used as a loading control. Error bars indicate standard error of the mean (SEM). For each cell line, two independent samples were used for replicate experiments ( $\geq 3$ ). (A) P16 protein expression in normal squamous esophageal (EPC2-hTERT) and EAC (OE19 and OE33) cell lines. (B) Total Rb expression in the cell lines. (C) Phosphorylated Rb (S795)/total Rb ratios in the normal and EAC cell lines. (D) Phosphorylated Rb (T356)/total Rb ratios in the normal and EAC cell lines.

Table III. Scoring percentages for phospho-Rb immunohistochemistry staining on BE tissue samples.

\begin{tabular}{llll}
\multicolumn{4}{c}{ Score } \\
\hline $0 / 1$ & 2 & 3 & 4
\end{tabular}

Phospho-Rb (T356)

$\begin{array}{lcccc}\mathrm{Sq} & 100 & 0 & 0 & 0 \\ \mathrm{EAC} & 10.5 & 5.26 & 47.4 & 36.8\end{array}$

Phospho-Rb (S795)

$\begin{array}{lcccc}\mathrm{Sq} & 100 & 0 & 0 & 0 \\ \mathrm{EAC} & 0 & 15.8 & 63.1 & 21.1\end{array}$

For phospho-Rb groups; scores 0,1 , no or very light staining in few nuclei; score 2, staining in few nuclei; score 3, staining in 33-50\% of nuclei; score 4 , clear staining in almost all nuclei.

Phospho-Rb immunohistochemistry staining in esophageal tissues. To validate and visualize the $\mathrm{Rb}$ phosphorylation as detected in the biopsy protein lysates we performed immunohistochemistry staining for Rb phosphorylated on the S795 and T356 groups on BE patient tissue samples. To this end
8 squamous esophageal and 19 EAC samples were stained. Overall, the stainings showed that the EAC tissues had a stronger phosphorylation on both the S795 and T356 groups than the squamous tissues (Fig. 5). To better (semi-)quantify these results we designed a scoring system for the staining performed, based on strength of staining and number of stained nuclei. The scoring confirmed that Rb phosphorylation on S795 and T356 was much more pronounced in EAC compared to squamous esophageal tissues (Table III). If we considered the two highest scoring categories as indicative of high $\mathrm{Rb}$ phosphorylation, than $84.2 \%$ of the EAC tissues showed a high phosphorylation of S795 and T356 compared to $0 \%$ of the squamous tissues. Another interesting result of the staining was that sequential EAC tissue sections stained for the two $\mathrm{Rb}$ groups did not show a complete overlap with respect to stained nuclei. This indicates that certain nuclei might have increased phosphorylation on one amino acid while lacking this increased phosphorylation on the other amino acid.

\section{Discussion}

Our explorative search into phosphorylation activity as a method to gain more insight into the pathogenesis of EAC confirmed that kinase activity is largely upregulated in this cancer. High phosphorylation levels have been described 

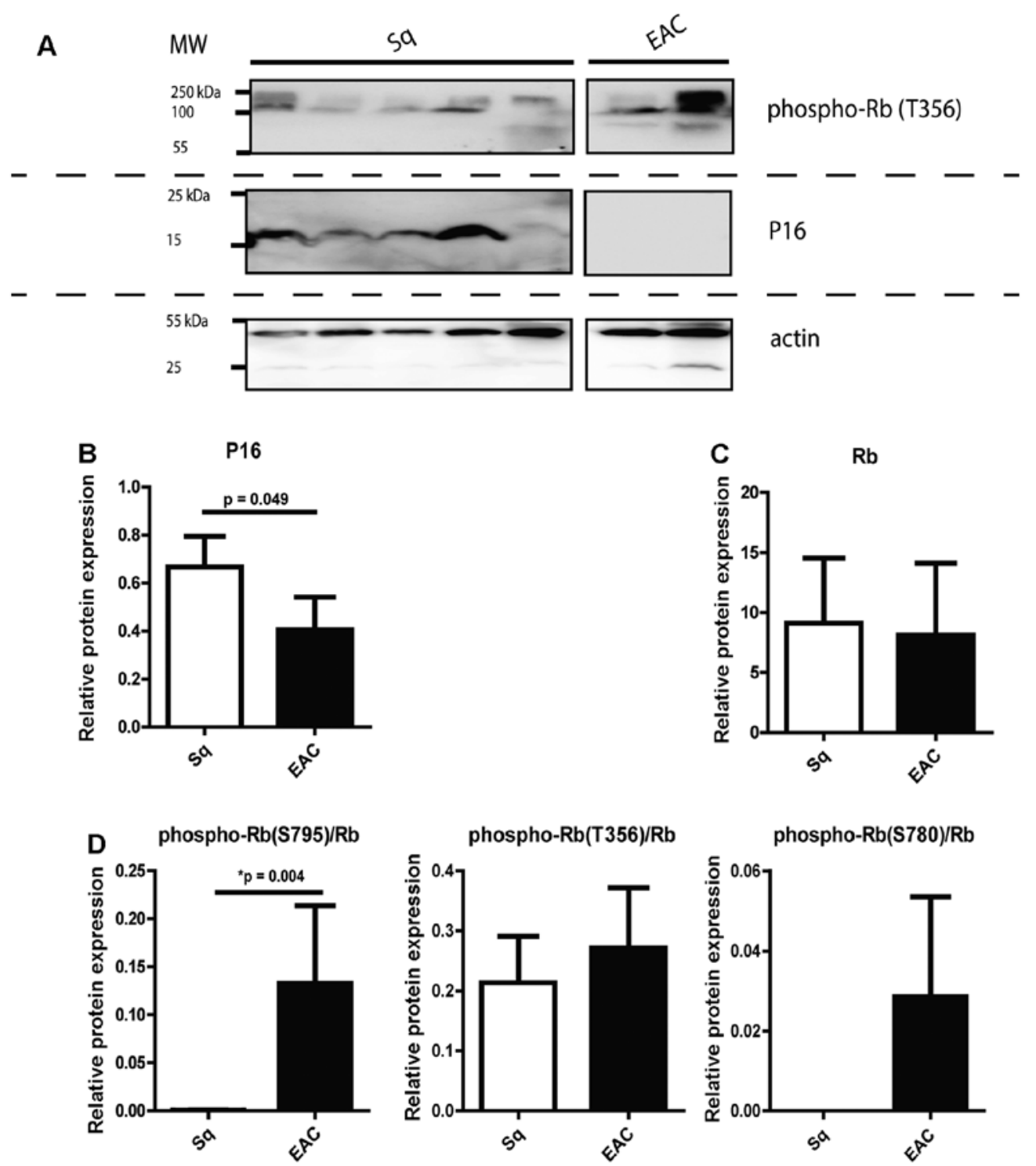

Figure 4. (A) Immunoblot for phospho-Rb(T356) and p16 protein in normal squamous and EAC biopsy lysates. (B) Quantification of immuno-blotting for p16 in esophageal biopsy lysates of normal squamous and EAC tissues. Samples are corrected for actin, used as a loading control. Error bars indicate SEM. (C) Total Rb expression in the biopsies. (D) Rb protein phosphorylation of the S795, T356 and S780 residues relative to total Rb in normal squamous and EAC tissues.

earlier in EAC as such our findings are in accordance with earlier studies $(27,28)$. To the best of our knowledge, however, this is the first report that describes the phosphorylation of specific Rb sites in EAC.

A list of the proteins for which phosphorylation was significantly different in EAC compared to normal esophageal tissues shows a broad range of molecules involved in varied processes (Table I). A large part of this list represents proteins involved in cytoskeletal structure organization and cell adhesion, but also proteins involved in immunity are represented. EAC is associated with chronic inflammation due to active reflux esophagitis (27-29). Aberrant phosphorylation related to the inflammatory process in EAC compared to normal squamous tissues falls in line with the literature (29-31). Tissue structure and inflammation related processes might be interesting candidates for molecular targets in EAC.

A pathway of interest that we investigated in the present study was the phosphorylation of the Rb protein in EAC. A 1.5-fold higher phosphorylation of $\mathrm{Rb}$ was observed by the kinase chip in EAC compared to normal esophageal tissues. This aberrant phosphorylation of $\mathrm{Rb}$ is likely due to aberrations of its upstream regulator, the p16. Aberrant p16 as detected through methylation, loss of heterozygosity and immunohistochemistry has been frequently observed in EAC pathogenesis (17-20). P16 has thus been a molecule of interest in EAC. In the present study we first investigated p16 status in EAC. There was a lower P16 protein expression in EAC tissues and cell lines compared to the normal squamous tissues (Figs. 3 and 4). On the genetic level P16 showed an aberrant status in EAC cell lines compared to a normal squamous esophageal cell line (Table II). The discrepancy of gains of the P16 locus seen in one EAC cell line combined with a lower P16 protein expression, is likely due to hypermethylation of the p16 gene promoter. A process frequently observed in $\operatorname{EAC}(17,18,20)$. We further found an inverse correlation between P16 expression and phosphorylation of $\mathrm{Rb}$ forms in EAC cell lines and biopsies (Figs. 3 and 4). Overall, EAC had lower P16 protein expression levels and increased phosphorylation levels of $\mathrm{Rb}$. 


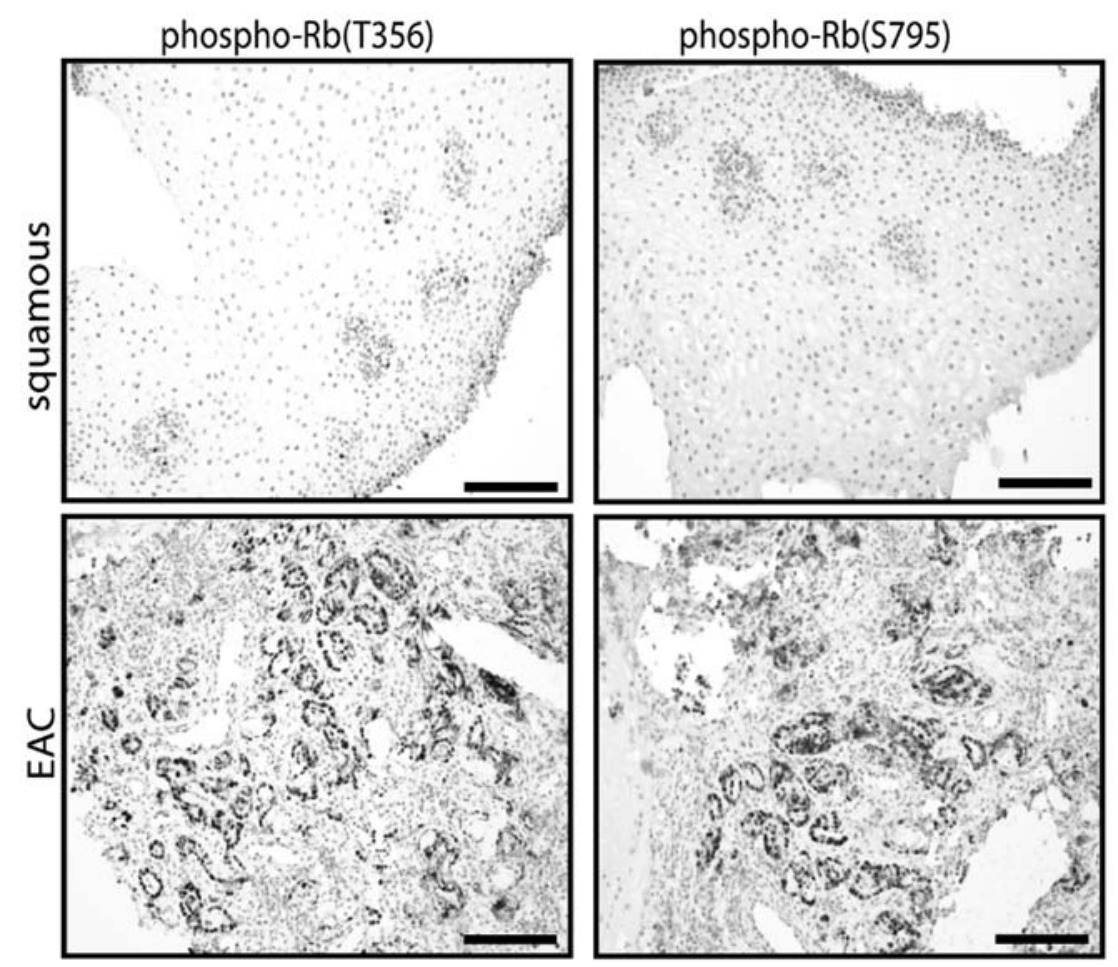

Figure 5. Immunohistochemistry stainings for phospho-Rb on squamous esophageal and EAC tissues. EAC tissues showed a higher nuclear expression of phospho-Rb. The scale bar indicates $200 \mu \mathrm{m}$.

The specific Rb phosphorylation sites of S795, S780 and T356, that we investigated all shared the same upstream cyclin dependent kinase 4 (CDK4). Phosphorylation at these amino acids leads to the detachment of $\mathrm{Rb}$ from its interacting proteins and stimulation of progression through the G1/S checkpoint (32-34). In the tissue lysates there was a higher rate of $\mathrm{Rb}$ phosphorylation on all three positions in EAC compared to the normal tissues. Overall, this effect seemed to be more pronounced for the S795 and S780, than the T356 site, and only for phosphorylation on the $\mathrm{S} 795$ position did this appear to be significant. However, this might be due to the relatively small number of samples investigated. Also in the immunohistochemistry stained slides the EAC tissues showed a higher expression of $\mathrm{Rb}$ phosphorylated on the S795 and T356 groups compared to the normal tissues. It is not clear how phosphorylation on the different sites would differ with respect to functioning. The S795 group lies within the $\mathrm{Rb}$ $\mathrm{C}$-terminal tail, believed to regulate binding of $\mathrm{Rb}$ interactors, such as E2F, to the Rb pocket domain $(35,36)$. The T356 group lies in the N-terminal interdomain linker region of $\mathrm{Rb}$ and is also believed to be involved in the regulation of binding of the E2F transcription factor $(37,38)$. However, as Rb interacts with a number of other different proteins through the different binding domains (35), it is possible that phosphorylation on the different $\mathrm{Rb}$ residues affect interactions with varied proteins and pathways. Notably, the immunohistochemistry staining patterns for phospho-Rb (S795) and phospho-Rb (T356) when comparing sequential slides differed indicating that specific nuclei might have an increased phosphorylation on a specific residue but not on the other. Future experiments should more closely examine whether specific phosphorylation on the different $\mathrm{Rb}$ residues might lead to differential results in EAC.
Also it might be of interest to investigate if there are specific $\mathrm{Rb}$ sites that are more phosphorylated in the earlier stages of EAC development, i.e. in BE.

Research has shown that inhibitors of kinases involved in cell proliferation might serve as anticancer therapeutics. With respect to $\mathrm{Rb}$ phosphorylation some research has been done on specific molecules affecting this process in EAC. For instance, Song et al (40) found that in cell lines of EAC and its premalignant stage of Barrett's esophagus, the green tea extract mixture of polyphenon E leads to an inhibition of proliferation and induction of apoptosis. This anti-proliferative function of the compound seemed to occur through the downregulation of the expression of cyclin D1, an upstream phosphorylating regulator of $\mathrm{Rb}$. Accordingly, this led to a lower expression of phospho-Rb in EAC and Barrett's cells (40). In another experiment the effects of flavopiridol, a pan-inhibitor of CDKs, on a mouse model of Barrett's and related EAC, were investigated (41). In this model esophagojejunostomy induced reflux combined with carcinogen exposure in a p27 null background, induced Barrett's development. These researchers found a significantly lower prevalence of BE and EAC in flavopiridol treated animals. This was combined with a lower expression of cyclin D1 in tumors of treated animals, with some tentative evidence for lower expression of phospho- $\mathrm{Rb}$ in treated mice (41). There are thus potential therapeutics that could be tested in EAC to further investigate how modulation of the specific phosphorylation of $\mathrm{Rb}$ could influence the disease.

In conclusion, overall phosphorylation activity is upregulated in EAC. This aberrant upregulation seems to be especially pronounced in processes related to inflammation and tissue structure organization. With respect to the p16-Rb pathway, $\mathrm{Rb}$ is significantly higher phosphorylated on the S795 
residue. This residue might be of interest as a putative target for treatment and further molecular insight in EAC and should be further investigated.

\section{References}

1. Pera M: Trends in incidence and prevalence of specialized intestinal metaplasia, Barrett's esophagus, and adenocarcinoma of the gastroesophageal junction. World J Surg 27: 999-1008, 2003.

2. Holmes RS and Vaughan TL: Epidemiology and pathogenesis of esophageal cancer. Semin Radiat Oncol 17: 2-9, 2007.

3. Lagergren $\mathbf{J}$ and Lagergren P: Recent developments in esophageal adenocarcinoma. CA Cancer J Clin 63: 232-248, 2013.

4. Edgren G, Adami HO, Weiderpass E and Nyrén O: A global assessment of the oesophageal adenocarcinoma epidemic. Gut 62: 1406-1414, 2013.

5. Hur C, Miller M, Kong CY, Dowling EC, Nattinger KJ, Dunn M and Feuer EJ: Trends in esophageal adenocarcinoma incidence and mortality. Cancer 119: 1149-1158, 2013.

6. Hoyo C, Cook MB, Kamangar F, Freedman ND, Whiteman DC, Bernstein L, Brown LM, Risch HA, Ye W, Sharp L, et al: Body mass index in relation to oesophageal and oesophagogastric junction adenocarcinomas: a pooled analysis from the International BEACON Consortium. Int J Epidemiol 41: 1706-1718, 2012.

7. Cossentino MJ and Wong RK: Barrett's esophagus and risk of esophageal adenocarcinoma. Semin Gastrointest Dis 14: 128-135, 2003.

8. Spechler SJ, Sharma P, Souza RF, Inadomi JM, Shaheen NJ; American Gastroenterological Association: American Gastroenterological Association technical review on the management of Barrett's esophagus. Gastroenterology 140: e18-e52, 2011.

9. Rubenstein JH and Taylor JB: Meta-analysis: the association of oesophageal adenocarcinoma with symptoms of gastro-oesophageal reflux. Aliment Pharmacol Ther 32: 1222-1227, 2010.

10. vanHagen $P$,Hulshof MC, vanLanschotJJ,Steyerberg EW, van Berge Henegouwen MI, Wijnhoven BP, Richel DJ, Nieuwenhuijzen GA, Hospers GA, Bonenkamp JJ, et al: Preoperative chemoradiotherapy for esophageal or junctional cancer. N Engl J Med 366: 2074-2084, 2012.

11. Baselga J, Carbonell X, Castañeda-Soto NJ, Clemens M, Green M, Harvey V, Morales S, Barton C and Ghahramani P: Phase II study of efficacy, safety, and pharmacokinetics of trastuzumab monotherapy administered on a 3-weekly schedule. J Clin Oncol 23: 2162-2171, 2005

12. Piccart-Gebhart MJ, Procter M, Leyland-Jones B, Goldhirsch A, Untch M, Smith I, Gianni L, Baselga J, Bell R, Jackisch C, et al: Trastuzumab after adjuvant chemotherapy in HER2-positive breast cancer. N Engl J Med 353: 1659-1672, 2005.

13. Gowryshankar A, Nagaraja V and Eslick GD: HER2 status in Barrett's esophagus \& esophageal cancer: a meta-analysis. J Gastrointest Oncol 5: 25-35, 2014

14. Bang YJ, van Cutsem E, Feyereislova A, Chung HC, Shen L, Sawaki A, Lordick F, Ohtsu A, Omuro Y, Satoh T, et al: Trastuzumab in combination with chemotherapy versus chemotherapy alone for treatment of HER2-positive advanced gastric or gastro-oesophageal junction cancer (ToGA): a phase 3, open-label, randomised controlled trial. Lancet 376: 687-697, 2010.

15. Sherr CJ and McCormick F: The RB and p53 pathways in cancer Cancer Cell 2: 103-112, 2002.

16. Rocco JW and Sidransky D: p16(MTS-1/CDKN2/INK4a) in Cancer Progression. Exp Cell Res 264: 42-55, 2001.

17. Vieth M, Schneider-Stock R, Rohrich K, May A, Ell C, Markwarth A, Roessner A, Stolte M and Tannapfel A: INK4aARF alterations in Barrett's epithelium, intraepithelial neoplasia and Barrett's adenocarcinoma. Virchows Arch 445: 135-141, 2004.

18. Hardiea LJ, Darnton SJ, Wallis YL, Chauhan A, Hainaut P, Wilda CP and Cassond AG: p16 expression in Barrett's esophagus and esophageal adenocarcinoma: association with genetic and epigenetic alterations. Cancer Lett 217: 221-230, 2005.

19. Wong DJ, Paulson TG, Prevo LJ, Galipeau PC, Longton G, Blount PL and Reid BJ: p16 ${ }^{\mathrm{INK} 4 \mathrm{a}}$ lesions are common, early abnormalities that undergo clonal expansion in Barrett's metaplastic epithelium. Cancer Res 61: 8284-8289, 2001.

20. Wang JS, Guo M, Montgomery EA, Thompson RE, Cosby H, Hicks L, Wang S, Herman JG and Canto MI: DNA promoter hypermethylation of p16 and APC predicts neoplastic progression in Barrett's esophagus. Am J Gastroenterol 104: 2153-2160, 2009.
21. Kaune KM, Neumann C, Hallermann C, Haller F, Schön MP and Middel P: Simultaneous aberrations of single CDKN2A network components and a high Rb phosphorylation status can differentiate subgroups of primary cutaneous B-cell lymphomas. Exp Dermatol 20: 331-335, 2011

22. Yuan J, Knorr J, Altmannsberger M, Goeckenjan G, Ahr A, Scharl A and Strebhardt K: Expression of p16 and lack of pRB in primary small cell lung cancer. J Pathol 189: 358-362, 1999.

23. Harada H, Nakagawa H, Oyama K, Takaoka M, Andl CD, Jacobmeier B, von Werder A, Enders GH, Opitz OG and Rustgi AK: Telomerase induces immortalization of human esophageal keratinocytes without p16INK4a inactivation. Mol Cancer Res 1: 729-738, 2003.

24. Rygiel AM, van Baal JW, Milano F, Wang KK, Ten Kate FJ, Fockens P, Rosmolen WD, Bergman JJ, Peppelenbosch MP and Krishnadath KK: Efficient automated assessment of genetic abnormalities detected by fluorescence in situ hybridization on brush cytology in a Barrett esophagus surveillance population. Cancer 109: 1980-1988, 2007.

25. Gu Y, Rosenblatt J and Morgan DO: Cell cycle regulation of CDK2 activity by phosphorylation of Thr160 and Tyr15. EMBO J 11: 3995-4005, 1992 .

26. Hong J, Resnick M, Behar J, Wang LJ, Wands J, DeLellis RA, Souza RF, Spechler SJ and Cao W: Acid-induced p16 hypermethylation contributes to development of esophageal adenocarcinoma via activation of NADPH oxidase NOX5-S. Am J Physiol Gastrointest Liver Physiol 299: G697-G706, 2010.

27. Kalinina T, Bockhorn M, Kaifi JT, Thieltges S, Güngör C, Effenberger KE, Strelow A, Reichelt U, Sauter G, Pantel K, et al: Insulin-like growth factor-1 receptor as a novel prognostic marker and its implication as a cotarget in the treatment of human adenocarcinoma of the esophagus. Int J Cancer 127: 1931-1940, 2010.

28. Hector A, Montgomery EA, Karikari C, Canto M, Dunbar KB, Wang JS, Feldmann G, Hong SM, Haffner MC, Meeker AK, et al: The Axl receptor tyrosine kinase is an adverse prognostic factor and a therapeutic target in esophageal adenocarcinoma. Cancer Biol Ther 10: 1009-1018, 2010.

29. Poehlmann A, Kuester D, Malfertheiner P, Guenther T and Roessner A: Inflammation and Barrett's carcinogenesis. Pathol Res Pract 208: 269-280, 2012.

30. Lao-Sirieix P and Fitzgerald RC: Role of the micro-environment in Barrett's carcinogenesis. Biochem Soc Trans 38: 327-330, 2010.

31. Abdel-Latif MM, Duggan S, Reynolds JV and Kelleher D: Inflammation and esophageal carcinogenesis. Curr Opin Pharmacol 9: 396-404, 2009.

32. Zarkowska T and Mittnacht S: Differential phosphorylation of the retinoblastoma protein by G1/S cyclin-dependent kinases. J Biol Chem 272: 12738-12746, 1997.

33. Kitagawa M, Higashi H, Jung HK, Suzuki-Takahashi I, Ikeda M, Tamai K, Kato J, Segawa K, Yoshida E, Nishimura S, et al: The consensus motif for phosphorylation by cyclin D1-Cdk4 is different from that for phosphorylation by cyclin A/E-Cdk2. EMBO J 15: 7060-7069, 1996

34. Connell-Crowley L, Harper JW and Goodrich DW: Cyclin D1/Cdk4 regulates retinoblastoma protein-mediated cell cycle arrest by site-specific phosphorylation. Mol Biol Cell 8: 287-301, 1997.

35. Dick FA: Structure-function analysis of the retinoblastoma tumor suppressor protein - is the whole a sum of its parts? Cell Div 2: 26, 2007.

36. Rubin SM, Gall AL, Zheng N and Pavletich NP: Structure of the $\mathrm{Rb}$ C-terminal domain bound to E2F1-DP1: a mechanism for phosphorylation-induced E2F release. Cell 123: 1093-1106, 2005.

37. Burke JR, Deshong AJ,Pelton JG and Rubin SM: Phosphorylationinduced conformational changes in the retinoblastoma protein inhibit E2F transactivation domain binding. J Biol Chem 285: 16286-16293, 2010.

38. Heilmann AM1 and Dyson NJ: Phosphorylation puts the $\mathrm{pRb}$ tumor suppressor into shape. Genes Dev 26: 1128-1130, 2012

40. Song S, Krishnan K, Liu K and Bresalier RS: Polyphenon E inhibits the growth of human Barrett's and aerodigestive adenocarcinoma cells by suppressing cyclin D1 expression. Clin Cancer Res 15: 622-631, 2009.

41. Lechpammer M, Xu X, Ellis FH, Bhattacharaya N, Shapiro GI and Loda M: Flavopiridol reduces malignant transformation of the esophageal mucosa in p27 knockout mice. Oncogene 24: $1683-1688,2005$. 